\title{
Rational Interpolation and Approximate Solution of Integral Equations
}

\author{
V. N. Rusak and N. V. Grib \\ Belarus State University, Minsk, Belarus \\ Belarus State Pedagogical University, Minsk, Belarus \\ Received March 16, 2011
}

\begin{abstract}
In the space of continuous periodic functions, we construct interpolation rational operators, use them to obtain quadrature formulas with positive coefficients which are exact on rational trigonometric functions of order $2 n$, and suggest an algorithm for an approximate solution of integral equations of the second kind. We estimate the accuracy of the approximate solution via the best trigonometric rational approximations to the kernel and the right-hand side of the integral equation.
\end{abstract}

DOI: $10.1134 /$ S001226611120115

Interpolation quadrature formulas designed for the integration of periodic functions well approximated by trigonometric polynomials were studied quite comprehensively $[1,2]$. They were used for the development of approximate methods for solving integral equations whose kernels and coefficients can be approximated by polynomials (e.g., see [3, p. 511]). In the present paper, we consider the interpolation of periodic functions by rational operators and its application to the construction of quadrature formulas of Gauss type and to the approximate solution of integral equations of the second kind.

Let $\left|\alpha_{k}\right|<1, k=1, \ldots, n$, and let

$$
\pi_{n}(z)=\prod_{k=1}^{n}\left(z-\alpha_{k}\right) /\left(1-\overline{\alpha_{k}} z\right)
$$

be the Blaschke product. Since $\left|\pi_{n}(z)\right|=1$ on the unit circle, we have

$$
\pi_{n}\left(e^{i \varphi}\right)=e^{i \Phi_{n}(\varphi)}
$$

where $\Phi_{n}(\varphi)=\arg \pi_{n}\left(e^{i \varphi}\right)$ and $0 \leq \Phi_{n}(0)=\arg \prod_{k=1}^{n}\left(1-\alpha_{k}\right) /\left(1-\overline{\alpha_{k}}\right)<2 \pi$. By using relation (1), one can readily find that

$$
\Phi_{n}^{\prime}(\varphi)=\sum_{k=1}^{n} \frac{1-\left|\alpha_{k}\right|^{2}}{\left(e^{i \varphi}-\alpha_{k}\right)\left(e^{-i \varphi}-\overline{\alpha_{k}}\right)}>0 .
$$

Consequently, as $\varphi$ varies from 0 to $2 \pi$, the argument $\Phi_{n}(\varphi)+\varphi / 2$ grows from $\Phi_{n}(0)$ to $\Phi_{n}(0)+(2 n+1) \pi$; consequently, the function $\sin \left(\Phi_{n}(\varphi)+\varphi / 2\right)$ has zeros at the points $\left\{\varphi_{k}\right\}$,

$$
0 \leq \varphi_{0}<\varphi_{1}<\cdots<\varphi_{2 n}<2 \pi .
$$

By using relation (1) and the Euler formulas, one can readily show that

$$
\begin{aligned}
\sin \left(\Phi_{n}(\varphi)+\frac{\varphi}{2}\right) & =\frac{e^{i \varphi / 2}}{2 i q_{n}(\varphi)} \prod_{k=1}^{n}\left(1-\alpha_{k} e^{-i \varphi}\right)\left(e^{i \varphi}-\alpha_{k}\right)-e^{-i \varphi / 2} \prod_{k=1}^{n}\left(1-\overline{\alpha_{k}} e^{i \varphi}\right)\left(e^{-i \varphi}-\overline{\alpha_{k}}\right) \\
& =\frac{p_{n+1 / 2}(\varphi)}{q_{n}(\varphi)}, \quad q_{n}(\varphi)=\prod_{k=1}^{n}\left(e^{-i \varphi}-\overline{\alpha_{k}}\right)\left(e^{i \varphi}-\alpha_{k}\right),
\end{aligned}
$$


where $p_{n+1 / 2}(\varphi)$ is a trigonometric polynomial of half-integer order $n+1 / 2$ with real coefficients and with zeros at the points $\left\{\varphi_{k}\right\}_{k=0}^{2 n}$. In this case, we have the expansion [4, p. 17]

$$
p_{n+1 / 2}(\varphi)=C \prod_{k=0}^{2 n} \sin \frac{\varphi-\varphi_{k}}{2} .
$$

Note that $\cos \left(\Phi_{n}(\varphi)+\varphi / 2\right)$ is a function of the form (3) as well with some trigonometric polynomial of half-integer order in the numerator and with the same denominator; in addition,

$$
\cos \left(\Phi_{n}\left(\varphi_{k}\right)+\frac{\varphi_{k}}{2}\right)=(-1)^{k} \cos \left(\Phi_{n}\left(\varphi_{0}\right)+\frac{\varphi_{0}}{2}\right) .
$$

By $Q_{n, 1}$ we denote the set $\left\{p_{n}(x) / q_{n}(x)\right\}$ of trigonometric rational functions of order $\leq n$ with given denominator $q_{n}(x)$; accordingly, by $Q_{n, 2}$ we denote the set $\left\{p_{2 n}(x) / q_{n}^{2}(x)\right\}$ of trigonometric rational functions of order $\leq 2 n$ with given denominator $q_{n}^{2}(x)$.

For any function $f(\varphi)$ in the space $C_{2 \pi}$ of continuous $2 \pi$-periodic functions, we construct an interpolation rational operator $L_{n}$ by setting

$$
L_{n}(\varphi, f)=\sum_{k=0}^{2 n} f\left(\varphi_{k}\right) l_{k, n}(\varphi), \quad l_{k, n}(\varphi)=\frac{\sin \left(\Phi_{n}(\varphi)+\varphi / 2\right) \cos \left(\Phi_{n}\left(\varphi_{k}\right)+\varphi_{k} / 2\right)}{\sin \left(\left(\varphi-\varphi_{k}\right) / 2\right)\left(1+2 \Phi_{n}^{\prime}\left(\varphi_{k}\right)\right)} .
$$

Lemma 1. The operator $L_{n}$ defined in (5)

1. takes each function $f \in C_{2 \pi}$ to a trigonometric rational function in $Q_{n, 1}$;

2. satisfies the conditions $L_{n}\left(\varphi_{k}, f\right)=f\left(\varphi_{k}\right), k=0, \ldots, 2 n$;

3. is exact on the set $Q_{n, 1}$.

Proof. By virtue of the representation (4), by dividing the half-integer trigonometric polynomial $p_{n+1 / 2}(\varphi)$ by $\sin \left(\left(\varphi-\varphi_{k}\right) / 2\right)$, we obtain a trigonometric polynomial $p_{k, n}(\varphi)$ of integer order $n$. By virtue of (3), the terms $f\left(\varphi_{k}\right) l_{k, n}(\varphi)$ differ from $p_{k, n}(\varphi) / q_{n}(\varphi)$ only by constants, i.e., are rational functions of order $\leq n$ with the same denominator $q_{n}(\varphi)$. Consequently, $\sum_{k=0}^{2 n} f\left(\varphi_{k}\right) l_{k, n}(\varphi) \in Q_{n, 1}$.

It follows from (4) and (5) that $l_{k, n}\left(\varphi_{j}\right)=0$ if $k \neq j$. But if $k=j$, then, by the l'Hôpital rule, we have

$$
l_{j, n}\left(\varphi_{j}\right)=\lim _{\varphi \rightarrow \varphi_{j}} l_{j, n}(\varphi)=\lim _{\varphi \rightarrow \varphi_{j}} \frac{\cos \left(\Phi_{n}(\varphi)+\varphi / 2\right)\left(\Phi_{n}^{\prime}(\varphi)+1 / 2\right) \cos \left(\Phi_{n}\left(\varphi_{j}\right)+\varphi_{j} / 2\right)}{\cos \left(\left(\varphi-\varphi_{j}\right) / 2\right)\left(1+2 \Phi_{n}^{\prime}\left(\varphi_{j}\right)\right) / 2}=1 .
$$

Then

$$
L_{n}\left(\varphi_{k}, f\right)=\sum_{k=0}^{2 n} f\left(\varphi_{j}\right) l_{j, n}\left(\varphi_{k}\right)=\sum_{k \neq j} 0+f\left(\varphi_{k}\right) \times 1=f\left(\varphi_{k}\right) .
$$

If the trigonometric rational function $r_{n}(\varphi)$ belongs to $Q_{n, 1}$, then the difference $r_{n}(\varphi)-L_{n}\left(\varphi, r_{n}\right)$ belongs to $Q_{n, 1}$ and is zero at the points $\varphi_{k}, k=0, \ldots, 2 n$; i.e., $r_{n}(\varphi)-L_{n}\left(\varphi, r_{n}\right)$ has $2 n+1$ zeros on $[0,2 \pi)$; therefore, $r_{n}(\varphi)-L_{n}\left(\varphi, r_{n}\right) \equiv 0$, since a trigonometric polynomial of order $\leq n$ has at most $2 n$ zeros in the half-strip $0 \leq \operatorname{Re} z<2 \pi$.

The norm in the space $C_{2 \pi}$ is given by the relation $\|f\|_{C_{2 \pi}}=\max \{|f(\varphi)|, \varphi \in[0,2 \pi]\}$. Note also that the interpolation operator (5) is treated as an operator mapping $C_{2 \pi}$ into $C_{2 \pi}$; thus (e.g., see [5, p. 201]),

$$
\left\|L_{n}(\varphi, f)\right\|_{C_{2 \pi}} \leq\left\|L_{n}\right\|\|f\|_{C_{2 \pi}}, \quad\left\|L_{n}\right\|=\max \left\{\sum_{k=0}^{2 n}\left|l_{k, n}(\varphi)\right|, \quad \varphi \in[0,2 \pi]\right\} .
$$

Using the interpolation operator (5), consider a quadrature formula for a function $f \in C_{2 \pi}$,

$$
\int_{0}^{2 \pi} f(\varphi) d \varphi \approx \sum_{k=0}^{2 n} A_{k} f\left(\varphi_{k}\right), \quad A_{k}=\int_{0}^{2 \pi} l_{k, n}(\varphi) d \varphi
$$


Lemma 2. The quadrature formula (7) has the following properties.

1. It is exact for any trigonometric rational function $r_{n} \in Q_{n, 1}$.

2. Its coefficients $A_{k}, k=0, \ldots, 2 n$, are positive; moreover,

$$
A_{k}=\frac{2 \pi}{2 \Phi_{n}^{\prime}\left(\varphi_{k}\right)+1}, \quad k=0, \ldots, 2 n .
$$

3. The relation $\sum_{k=0}^{2 n} A_{k}=2 \pi$ is satisfied.

Proof. If $r_{n} \in Q_{n, 1}$, then, by Lemma $1, r_{n}(\varphi)=L_{n}\left(\varphi, r_{n}\right)$; by integrating this relation, we obtain

$$
\int_{0}^{2 \pi} r_{n}(\varphi) d \varphi=\int_{0}^{2 \pi} L_{n}\left(\varphi, r_{n}\right) d \varphi=\sum_{k=0}^{2 n} r_{n}\left(\varphi_{k}\right) \int_{0}^{2 \pi} l_{k, n}(\varphi) d \varphi=\sum_{k=0}^{2 n} A_{k} r_{n}\left(\varphi_{k}\right),
$$

which completes the proof of the first property.

One should evaluate the integral

$$
I_{k}\left(e^{i \varphi_{k}}\right)=\int_{0}^{2 \pi} \frac{\sin \left(\Phi_{n}(\varphi)+\varphi / 2\right)}{\sin \left(\left(\varphi-\varphi_{k}\right) / 2\right)} d \varphi=e^{i \varphi_{k} / 2} \int_{0}^{2 \pi} \frac{e^{i\left(\Phi_{n}(\varphi)+\varphi\right)}-e^{-i \Phi_{n}(\varphi)}}{e^{i \varphi}-e^{i \varphi_{k}}} d \varphi .
$$

Using relation (1) for $|z|<1$, consider the integral

$$
\begin{aligned}
\widetilde{I}_{k}(z) & =e^{i \varphi_{k} / 2} \int_{0}^{2 \pi} \frac{e^{i\left(\Phi_{n}(\varphi)+\varphi\right)}-e^{-i \Phi_{n}(\varphi)}}{e^{i \varphi}-z} d \varphi=e^{i \varphi_{k} / 2} \int_{0}^{2 \pi} \frac{\pi_{n}\left(e^{i \varphi}\right) e^{i \varphi}-\pi_{n}\left(e^{i \varphi}\right)^{-1}}{e^{i \varphi}-z} \frac{d e^{i \varphi}}{i e^{i \varphi}} \\
& =\frac{e^{i \varphi_{k} / 2}}{i} \int_{|z|=1} \frac{\xi \pi_{n}(\xi)-\pi_{n}(\xi)^{-1}}{(\xi-z) \xi} d \xi .
\end{aligned}
$$

Since, by the Cauchy integral formula,

$$
\int_{|z|=1}(\xi-z)^{-1} \pi_{n}(\xi) d \xi=2 \pi i \pi_{n}(z)
$$

and the function $\left((\xi-z) \xi \pi_{n}(\xi)\right)^{-1}$ has no singular points in the domain $|\xi|>1$ and has a secondorder zero at infinity, it follows from (8) that $\widetilde{I}_{k}(z)=2 \pi e^{i \varphi_{k} / 2} \pi_{n}(z)$. Obviously,

$$
I_{k}\left(e^{i \varphi_{k}}\right)=\lim _{z \rightarrow e^{i \varphi_{k}}} \widetilde{I}_{k}(z)=2 \pi e^{i \varphi_{k} / 2} \pi_{n}\left(e^{i \varphi_{k}}\right)=2 \pi e^{i\left(\Phi_{n}\left(\varphi_{k}\right)+\varphi_{k} / 2\right)}=2 \pi \cos \left(\Phi_{n}\left(\varphi_{k}\right)+\varphi_{k} / 2\right) .
$$

This relation, together with (5) and (7), implies that

$$
A_{k}=\frac{\cos \left(\Phi_{n}\left(\varphi_{k}\right)+\varphi_{k} / 2\right)}{2 \Phi_{n}^{\prime}\left(\varphi_{k}\right)+1} \int_{0}^{2 \pi} \frac{\sin \left(\Phi_{n}(\varphi)+\varphi_{k} / 2\right)}{\sin \left(\left(\varphi-\varphi_{k}\right) / 2\right)} d \varphi=\frac{2 \pi}{2 \Phi_{n}^{\prime}\left(\varphi_{k}\right)+1}, \quad k=0, \ldots, 2 n
$$

the coefficients $A_{k}$ are positive by virtue of inequality (2).

To prove property 3 , it suffices to note that $r_{n}(x) \equiv 1 \in Q_{n, 1}$, and, by virtue of the exactness of the quadrature formula on $Q_{n, 1}$, we obtain

$$
\sum_{k=0}^{2 n} A_{k}=\int_{0}^{2 \pi} d \varphi=2 \pi
$$

The proof of Lemma 2 is complete.

DIFFERENTIAL EQUATIONS $\quad$ Vol. 48 No. 22012 
For a function $f \in C_{2 \pi}$, we define the best uniform approximation by trigonometric rational functions in $Q_{n, 1}$ and $Q_{n, 2}$ by setting

$$
R_{n}^{T}(f)=\inf \left(\left\|f-r_{n}\right\|, r_{n} \in Q_{n, 1}\right), \quad R_{2 n}^{T}(f)=\inf \left(\left\|f-r_{2 n}\right\|, r_{2 n} \in Q_{n, 2}\right) .
$$

Theorem 1. The quadrature formula (7) is exact for rational functions in the set $Q_{n, 2}$, and its accuracy can be estimated as

$$
\left|\int_{0}^{2 \pi} f(\varphi) d \varphi-\sum_{k=0}^{2 n} A_{k} f\left(\varphi_{k}\right)\right| \leq 4 \pi R_{2 n}^{T}(f)
$$

Proof. Let us show that each trigonometric rational function $r_{2 n} \in Q_{n, 2}$ can be represented in the form

$$
r_{2 n}(\varphi)=\sin \left(\Phi_{n}(\varphi)+\frac{\varphi}{2}\right) \frac{p_{n-1 / 2}(\varphi)}{q_{n}(\varphi)}+\frac{p_{n}(\varphi)}{q_{n}(\varphi)}
$$

where $p_{n-1 / 2}(\varphi)$ is a trigonometric polynomial of half-integer order and the rational function $p_{n}(\varphi) / q_{n}(\varphi)$ belongs to $Q_{n, 1}$.

Indeed, let $L_{n}\left(\varphi, r_{2 n}\right)$ be the value of the interpolation operator for the function $r_{2 n}$; then $L_{n}\left(\varphi, r_{2 n}\right)=r_{n}(\varphi)=p_{n}(\varphi) / q_{n}(\varphi)$, and the difference $r_{2 n}(\varphi)-p_{n}(\varphi) / q_{n}(\varphi)$ is zero at the points $\left\{\varphi_{k}\right\}_{k=0}^{2 n}$; therefore,

$$
r_{2 n}(\varphi)-\frac{p_{n}(\varphi)}{q_{n}(\varphi)}=\sin \left(\Phi_{n}(\varphi)+\frac{\varphi}{2}\right) \frac{p_{n-1 / 2}(\varphi)}{q_{n}(\varphi)}
$$

which is equivalent to relation (10).

Let us now show that

$$
\int_{0}^{2 \pi} \sin \left(\Phi_{n}(\varphi)+\frac{\varphi}{2}\right) \frac{p_{n-1 / 2}(\varphi)}{q_{n}(\varphi)} d \varphi=0 .
$$

Since a trigonometric polynomial of half-integer order can be represented in the form

$$
t_{n-1 / 2}(\varphi)=\sum_{j=1}^{n}\left(a_{j} e^{i(j-1 / 2) \varphi}+b_{j} e^{-i(j-1 / 2) \varphi}\right),
$$

we find that relation (11) follows from the relations

$$
I_{j}=\int_{0}^{2 \pi} \sin \left(\Phi_{n}(\varphi)+\frac{\varphi}{2}\right) \frac{e^{i(j-1 / 2) \varphi}}{q_{n}(\varphi)} d \varphi=0, \quad j=1, \ldots, n,
$$

which should be proved. By using the Euler formulas again and by passing to complex variables, we obtain

$$
\begin{aligned}
I_{j} & =\frac{1}{2 i} \int_{0}^{2 \pi}\left(e^{i\left(\Phi_{n}(\varphi)+\varphi / 2\right)}-e^{-i\left(\Phi_{n}(\varphi)+\varphi / 2\right)}\right) \frac{e^{i(j-1 / 2) \varphi}}{q_{n}(\varphi)} d \varphi \\
& =\frac{1}{2 i} \int_{0}^{2 \pi} \frac{\pi_{n}\left(e^{i \varphi}\right) e^{i \varphi / 2}-\pi_{n}\left(e^{i \varphi}\right)^{-1} e^{-i \varphi / 2}}{\prod_{k=1}^{n}\left(e^{i \varphi}-\alpha_{k}\right)\left(e^{-i \varphi}-\overline{\alpha_{k}}\right)} e^{i(j-1 / 2) \varphi} d \varphi \\
& =-\frac{1}{2} \int_{|\xi|=1} \frac{\xi^{n+j-1}}{\prod_{k=1}^{n}\left(1-\overline{\alpha_{k}} \xi\right)^{2}} d \xi+\frac{1}{2} \int_{|\xi|=1} \frac{\xi^{n+j-2}}{\prod_{k=1}^{n}\left(\xi-\alpha_{k}\right)^{2}} d \xi=0,
\end{aligned}
$$


because the integrand in the first integral on the right-hand side is analytic in $|z| \leq 1$, and that in the second integral is analytic in $|\xi| \geq 1$ and has a second-order zero at the point at infinity.

It follows from relations (10) and (11) and Lemma 2 that

$$
\int_{0}^{2 \pi} r_{2 n}(\varphi) d \varphi=\int_{0}^{2 \pi} \frac{p_{n}(\varphi)}{q_{n}(\varphi)} d \varphi=\int_{0}^{2 \pi} r_{n}(\varphi) d \varphi=\sum_{k=0}^{2 n} A_{k} r_{n}\left(\varphi_{k}\right)=\sum_{k=0}^{2 n} A_{k} r_{2 n}\left(\varphi_{k}\right) ;
$$

i.e., the quadrature formula (7) is exact on the set $Q_{n, 2}$; in other words, formula (7) is a quadrature formula of the Gauss type.

Let $r_{2 n}^{*}(\varphi)$ be a trigonometric rational function of the best approximation for the function $f \in C_{2 \pi}, r_{2 n}^{*}(\varphi) \in Q_{n, 2} ;$ i.e., the relation

$$
\left\|f-r_{2 n}^{*}\right\|_{C_{2 \pi}}=R_{2 n}^{T}(f)
$$

holds. For the accuracy of the quadrature formula, we have

$$
\begin{aligned}
\left|\int_{0}^{2 \pi} f(\varphi) d \varphi-\sum_{k=0}^{2 n} A_{k} f\left(\varphi_{k}\right)\right| & =\left|\int_{0}^{2 \pi}\left(f(\varphi)-r_{2 n}^{*}(\varphi)\right) d \varphi+\sum_{k=0}^{2 n} A_{k}\left(r_{2 n}^{*}\left(\varphi_{k}\right)-f\left(\varphi_{k}\right)\right)\right| \\
& \leq \int_{0}^{2 \pi}\left|f(\varphi)-r_{2 n}^{*}(\varphi)\right| d \varphi+\sum_{k=0}^{2 n} A_{k}\left|r_{2 n}^{*}\left(\varphi_{k}\right)-f\left(\varphi_{k}\right)\right| \leq 4 \pi R_{2 n}^{T}(f) .
\end{aligned}
$$

The proof of Theorem 1 is complete.

Note that $R_{n}^{T}(f) \geq R_{2 n}^{T}(f)$, and inequality (9) remains valid if $R_{2 n}^{T}(f)$ is replaced by $R_{n}^{T}(f)$.

Now consider the integral equation

$$
x(s)-\lambda \int_{0}^{2 \pi} h(s, t) x(t) d t=y(s)
$$

where $y(s) \in C_{2 \pi}$ is a known function and the kernel $h(s, t)$ has period $2 \pi$ with respect to each variable and is jointly continuous. It is known that, for small $|\lambda|$, there exists a unique solution of Eq. (13), which is a continuous periodic function (e.g., see [6, p. 109]). By replacing the integral on the left-hand side in relation (13) by a quadrature formula and by requiring the validity of that relation only at the node points $\left\{\varphi_{j}\right\}_{j=0}^{2 n}$, we obtain the system of linear algebraic equations

$$
z_{j}-\lambda \sum_{k=0}^{2 n} A_{k} h\left(\varphi_{j}, \varphi_{k}\right) z_{k}=y\left(\varphi_{j}\right), \quad j=0, \ldots, 2 n,
$$

whose solution provides the approximate values of the solution of Eq. (13) at the node points. We define an approximate solution for all values of $s$ as a rational function defined by the interpolation operator (5) at $\left\{z_{k}\right\}_{k=0}^{2 n}$,

$$
z(s)=\sum_{k=0}^{2 n} z_{k} l_{k, n}(s) .
$$

It is also convenient to rewrite system (14) in the operator form

$$
\widetilde{K}\left[\begin{array}{c}
z_{0} \\
z_{1} \\
\ldots \\
z_{2 n}
\end{array}\right]=\left[\begin{array}{c}
y\left(\varphi_{0}\right) \\
y\left(\varphi_{1}\right) \\
\ldots \\
y\left(\varphi_{2 n}\right)
\end{array}\right] \Leftrightarrow\left[\begin{array}{c}
z_{0} \\
z_{1} \\
\ldots \\
z_{2 n}
\end{array}\right]=\widetilde{K}^{-1}\left[\begin{array}{c}
y\left(\varphi_{0}\right) \\
y\left(\varphi_{1}\right) \\
\ldots \\
y\left(\varphi_{2 n}\right)
\end{array}\right],
$$

DIFFERENTIAL EQUATIONS Vol. 48 No. $2 \quad 2012$

(Reg. No. 211, 9.3.2012) 
where $\widetilde{K}$ is a linear operator on the space $R^{2 n+1}$ and $\widetilde{K}^{-1}$ is the corresponding inverse operator on the same space.

The integral equation (13) is also represented in the operator form $K x=y$, where $K$ is a linear operator on the space $C_{2 \pi}$ and $\|K\|$ is the norm of it treated as an operator mapping $C_{2 \pi}$ into $C_{2 \pi}$.

We assume that the kernel $h(s, t)$ can be approximated by rational functions in the set $Q_{n, 1}$ with respect to each variable; i.e., there exist functions

$$
\begin{aligned}
& h_{1}(s, t)=\frac{1}{q_{n}(t)}\left(\frac{a_{0}^{\prime}(s)}{2}+\sum_{k=1}^{n} a_{k}^{\prime}(s) \cos k t+b_{k}^{\prime}(s) \sin k t\right), \\
& h_{2}(s, t)=\frac{1}{q_{n}(s)}\left(\frac{a_{0}^{\prime \prime}(t)}{2}+\sum_{k=1}^{n} a_{k}^{\prime \prime}(t) \cos k s+b_{k}^{\prime \prime}(t) \sin k s\right),
\end{aligned}
$$

with continuous $2 \pi$-periodic coefficients and these functions satisfy the inequalities

$$
\left|h(s, t)-h_{1}(s, t)\right| \leq R_{\infty, n}^{T}(h), \quad\left|h(s, t)-h_{2}(s, t)\right| \leq R_{n, \infty}^{T}(h), \quad 0 \leq s, t \leq 2 \pi .
$$

Theorem 2. If $x(s)$ is the exact solution of Eq. (13) whose kernels satisfy condition (18) and $z(s) \in Q_{n, 1}$ is an approximate solution found from Eqs. (14) and (15), then the following estimate holds:

$$
\begin{aligned}
\|x(s)-z(s)\|_{C_{2 \pi} \leq} \leq & \left(\left\|L_{n}\right\|\left\|\widetilde{K}^{-1}\right\|\|K\|+1\right)\left(2 \pi|\lambda| R_{n, \infty}^{T}(h)\|x\|+R_{n}^{T}(y)\right) \\
& +4 \pi\left\|L_{n}\right\||\lambda|\left\|\widetilde{K}^{-1}\right\| R_{\infty, n}^{T}(h)\left(R_{n}^{T}(y)+\|x\|\left(1+2 \pi|\lambda| R_{n, \infty}^{T}(h)\right)\right) .
\end{aligned}
$$

Proof. First, we approximate the exact solution by the rational function

$$
\bar{x}(s)=\lambda \int_{0}^{2 \pi} h_{2}(s, t) x(t) d t+\bar{y}(s),
$$

where $\bar{y}(s) \in Q_{n, 1}$ is the rational function of the best approximation to $y(s)$; therefore, $\|y(s)-\bar{y}(s)\|=R_{n}^{T}(y)$. By subtracting relation (20) from (13) and by taking into account conditions (18), we obtain

$$
\|x(s)-\bar{x}(s)\|_{C_{2 \pi}}=\left\|\lambda \int_{0}^{2 \pi}\left(h(s, t)-h_{2}(s, t)\right) x(t) d t+y(s)-\bar{y}(s)\right\| \leq 2 \pi|\lambda| R_{n, \infty}^{T}(h)\|x\|+R_{n}^{T}(y) .
$$

Now we define a rational function $\widetilde{x}(s)$ from the nodal values $\left\{\varphi_{j}\right\}_{j=0}^{2 n}$ by setting [with regard of (16)]

$$
\left[\begin{array}{c}
\widetilde{x}\left(\varphi_{0}\right) \\
\widetilde{x}\left(\varphi_{1}\right) \\
\cdots \\
\widetilde{x}\left(\varphi_{2 n}\right)
\end{array}\right]=\widetilde{K}^{-1}\left[\begin{array}{c}
u_{0} \\
u_{1} \\
\ldots \\
u_{2 n}
\end{array}\right], \quad u_{j}=\bar{x}\left(\varphi_{j}\right)-\lambda \int_{0}^{2 \pi} h\left(\varphi_{j}, t\right) \bar{x}(t) d t, \quad j=0, \ldots, 2 n
$$

By virtue of relations (14) and (16), the values of the rational function $\bar{x}(s)$ can be determined in the form

$$
\left[\begin{array}{c}
\bar{x}\left(\varphi_{0}\right) \\
\bar{x}\left(\varphi_{1}\right) \\
\ldots \\
\bar{x}\left(\varphi_{2 n}\right)
\end{array}\right]=\widetilde{K}^{-1}\left[\begin{array}{c}
v_{0} \\
v_{1} \\
\ldots \\
v_{2 n}
\end{array}\right], \quad v_{j}=\bar{x}\left(\varphi_{j}\right)-\lambda \sum_{k=0}^{2 n} A_{k} h\left(\varphi_{j}, \varphi_{k}\right) \bar{x}\left(\varphi_{k}\right), \quad j=0, \ldots, 2 n .
$$


By subtracting relations (23) from (22) and by estimating the values of the operator $R^{2 n+1}$ via its norm, we obtain the inequality

$$
\max _{0 \leq j \leq 2 n}\left|\bar{x}\left(\varphi_{j}\right)-\widetilde{x}\left(\varphi_{j}\right)\right| \leq\left\|\widetilde{K}^{-1}\right\| \max _{0 \leq j \leq 2 n}\left|\lambda \int_{0}^{2 \pi} h\left(\varphi_{j}, t\right) \bar{x}(t) d t-\lambda \sum_{k=0}^{2 n} A_{k} h\left(\varphi_{j}, \varphi_{k}\right) \bar{x}\left(\varphi_{k}\right)\right| .
$$

By using the kernel $h_{1}(s, t)$, which, by virtue of the representation (17), is a rational function of $t$ in the set $Q_{n, 1}$, and by using the fact that, for any $s$, the product $h_{1}(s, t) \bar{x}(t)$ is a rational function in $Q_{n, 2}$, from Theorem 1, we obtain the relation

$$
\int_{0}^{2 \pi} h_{1}\left(\varphi_{j}, t\right) \bar{x}(t) d t=\sum_{k=0}^{2 n} A_{k} h_{1}\left(\varphi_{j}, \varphi_{k}\right) \bar{x}\left(\varphi_{k}\right)
$$

It follows from relations (24), (25), (18), Lemma 2, and inequality (21) that

$$
\begin{aligned}
& \max _{0 \leq j \leq 2 n}\left|\bar{x}\left(\varphi_{j}\right)-\widetilde{x}\left(\varphi_{j}\right)\right| \\
& \quad \leq\left\|\widetilde{K}^{-1}\right\| \max _{0 \leq j \leq 2 n}\left|\lambda \int_{0}^{2 \pi}\left(h\left(\varphi_{j}, t\right)-h_{1}\left(\varphi_{j}, t\right)\right) \bar{x}(t) d t+\lambda \sum_{k=0}^{2 n} A_{k}\left(h_{1}\left(\varphi_{j}, \varphi_{k}\right)-h\left(\varphi_{j}, \varphi_{k}\right)\right) \bar{x}\left(\varphi_{k}\right)\right| \\
& \quad \leq 4 \pi\left\|\widetilde{K}^{-1}\right\||\lambda|\|\bar{x}\| R_{\infty, n}^{T}(h) \leq 4 \pi\left\|\widetilde{K}^{-1}\right\||\lambda| R_{\infty, n}^{T}(h)\left(\|x\|\left(1+2 \pi|\lambda| R_{n, \infty}^{T}(h)\right)+R_{n}^{T}(y)\right) .
\end{aligned}
$$

Then, by taking into account (6), for the uniform norm, we obtain

$$
\|\bar{x}(\varphi)-\widetilde{x}(\varphi)\|_{C_{2 \pi}} \leq 4 \pi\left\|L_{n}\right\|\left\|\widetilde{K}^{-1}\right\||\lambda| R_{\infty, n}^{T}(h)\left(\|x\|\left(1+2 \pi|\lambda| R_{n, \infty}^{T}(h)\right)+R_{n}^{T}(y)\right) .
$$

If $\left\{z_{j}\right\}_{j=0}^{2 n}$ is a solution of system (14), then, by virtue of Eqs. (13) and (16), we have

$$
\left[\begin{array}{c}
z_{0} \\
z_{1} \\
\cdots \\
z_{2 n}
\end{array}\right]=\widetilde{K}^{-1}\left[\begin{array}{c}
y\left(\varphi_{0}\right) \\
y\left(\varphi_{1}\right) \\
\cdots \\
y\left(\varphi_{2 n}\right)
\end{array}\right], \quad y\left(\varphi_{j}\right)=x\left(\varphi_{j}\right)-\lambda \int_{0}^{2 \pi} h\left(\varphi_{j}, t\right) x(t) d t, \quad j=0, \ldots, 2 n,
$$

where $x(t)$ is an exact solution of the integral equation.

It follows from relations (22), (28), (13), and (21) that

$$
\begin{aligned}
\max _{0 \leq j \leq 2 n}\left|\widetilde{x}\left(\varphi_{j}\right)-z_{j}\right| & \leq\left\|\widetilde{K}^{-1}\right\| \max _{0 \leq j \leq 2 n}\left|\bar{x}\left(\varphi_{j}\right)-x\left(\varphi_{j}\right)+\lambda \int_{0}^{2 \pi} h\left(\varphi_{j}, t\right)(x(t)-\bar{x}(t)) d t\right| \\
& \leq\left\|\widetilde{K}^{-1}\right\|\|K\|\|x(s)-\bar{x}(s)\| \leq\left\|\widetilde{K}^{-1}\right\|\|K\|\left(2 \pi|\lambda| R_{n, \infty}^{T}(h)\|x\|+R_{n}^{T}(y)\right) ;
\end{aligned}
$$

therefore, by virtue of $(6)$, for the uniform norm $\|\widetilde{x}(s)-z(s)\|$ [see (15)], we obtain the estimate

$$
\|\widetilde{x}(s)-z(s)\| \leq\left\|L_{n}\right\|\left\|\widetilde{K}^{-1}\right\|\|K\|\left(2 \pi|\lambda| R_{n, \infty}^{T}(h)\|x\|+R_{n}^{T}(y)\right) .
$$

By combining the estimates (21), 927), and (29), as a result, we get

$$
\begin{aligned}
\|x(s)-z(s)\| \leq & \|x(s)-\bar{x}(s)\|+\|\bar{x}(s)-\widetilde{x}(s)\|+\|\widetilde{x}(s)-z(s)\| \\
\leq & \left(2 \pi|\lambda| R_{n, \infty}^{T}(h)\|x\|+R_{n}^{T}(y)\right)\left(1+\left\|L_{n}\right\|\left\|\widetilde{K}^{-1}\right\|\|K\|\right) \\
& +4 \pi\left\|L_{n}\right\|\left\|\widetilde{K}^{-1}\right\||\lambda| R_{\infty, n}^{T}(h)\left(\|x\|\left(1+2 \pi|\lambda| R_{n, \infty}^{T}(h)\right)+R_{n}^{T}(y)\right),
\end{aligned}
$$

which coincides with the estimate (19).

DIFFERENTIAL EQUATIONS $\quad$ Vol. 48 No. $2 \quad 2012$ 
Remark. All quantities occurring on the right-hand side in the estimate (19) are bounded; this fails only for the norm $\left\|L_{n}\right\|$ of the interpolation operator, which, in a number of important cases, has logarithmic growth with respect to $n$. This right-hand side depends essentially on the parameters $\left\{\alpha_{k}\right\}_{k=1}^{n}$, which are chosen depending on properties of the kernel $h(s, t)$ and the righthand side $y(s)$ of the integral equation. If, for integer values of $n$ and for a positive $\alpha$, there exist sets $\left\{\alpha_{k}\right\}_{k=0}^{n}$ such that

$$
R_{\infty, n}^{T}(h)=O\left(\frac{1}{n^{\alpha}}\right), \quad R_{n, \infty}^{T}(h)=O\left(\frac{1}{n^{\alpha}}\right), \quad R_{n}^{T}(y)=O\left(\frac{1}{n^{\alpha}}\right)
$$

then it readily follows from Theorem 2 that the accuracy $\|x(s)-z(s)\|_{C_{2 \pi}}$ satisfies the order estimate

$$
\|x(s)-z(s)\|_{C_{2 \pi}}=O\left(\frac{\left\|L_{n}\right\|}{n^{\alpha}}\right) .
$$

In conclusion, we note that the rational approximation was used in [7] for the estimate of the accuracy of an approximate solution of a characteristic singular integral equation.

\section{REFERENCES}

1. Nikol'skii, S.M., Kvadraturnye formuly (Quadrature Formulas), Moscow, 1974.

2. Turetskii, A.Kh., On Quadrature Formulas with an Even Number of Nodes, Which Are Exact for Trigonometric Polynomials, Dokl. Akad. Navuk BSSR, 1960, vol. 4, no. 9, p. 365.

3. Kantorovich, L.V. and Akilov, G.P., Funktsional'nyi analiz v normirovannykh prostranstvakh (Functional Analysis in Normed Spaces), Moscow: Gosudarstv. Izdat. Fiz.-Mat. Lit., 1959.

4. Turetskii, A.Kh., Teoriya interpolirovaniya $v$ zadachakh (Theory of Interpolation in Problems), Minsk, 1968.

5. Korovkin, P.P., Lineinye operatory i teoriya priblizhenii (Linear Operators and Approximation Theory), Moscow, 1959.

6. Kantorovich, L.V. and Krylov, V.I., Priblizhennye metody vysshego analiza (Approximated Methods of Higher Analysis), Leningrad; Moscow, 1941.

7. Mitenkov, V.I. and Rusak, V.N., An Error Estimate for an Approximation of a Characteristic Singular Integral Equation, Differ. Uravn., 2001, vol. 37, no. 3, pp. 410-414. 